\title{
BMJ Open Protocol for a feasibility study: a brief self-compassion intervention for adolescents with type 1 diabetes and disordered eating
}

\author{
Anna L Boggiss (D) , ${ }^{1}$ Nathan S Consedine (D) , ${ }^{1}$ Craig Jefferies, ${ }^{2}$ Karen Bluth, ${ }^{3}$ \\ Paul L Hofman, ${ }^{4}$ Anna S Serlachius ${ }^{1}$
}

To cite: Boggiss AL,

Consedine NS, Jefferies C, et al. Protocol for a feasibility study: a brief self-compassion intervention for adolescents with type 1 diabetes and disordered eating. BMJ Open 2020;10:e034452. doi:10.1136/ bmjopen-2019-034452

- Prepublication history for this paper is available online. To view these files, please visit the journal online (http://dx.doi. org/10.1136/bmjopen-2019034452).

Received 20 September 2019 Revised 19 December 2019 Accepted 20 January 2020

Check for updates

(C) Author(s) (or their employer(s)) 2020. Re-use permitted under CC BY-NC. No commercial re-use. See rights and permissions. Published by BMJ.

${ }^{1}$ Department of Psychological Medicine, The University of

Auckland Faculty of Medical and Health Sciences, Auckland, New Zealand

${ }^{2}$ Starship Children's Health, Auckland City Hospital,

Auckland, New Zealand

${ }^{3}$ Department of Psychiatry, University of North Carolina at Chapel Hill, Chapel Hill, North Carolina, USA

${ }^{4}$ The Liggins Institute, The University of Auckland, Auckland, New Zealand

Correspondence to Anna L Boggiss;

abog579@aucklanduni.ac.nz

\section{ABSTRACT}

Introduction Adolescents with type 1 diabetes are at a higher risk of developing psychiatric disorders, particularly eating disorders, compared with their healthy peers. In turn, this increases the risk for sub-optimal glycaemic control and life-threatening diabetes-related complications. Despite these increased risks, standard diabetes care does not routinely provide psychological support to help prevent or reduce mental health risks. There is an urgent need to develop 'clinically usable' psychosocial interventions that are acceptable to patients and can be realistically integrated into clinical care. This study aims to examine the feasibility and acceptability of a brief self-compassion intervention for adolescents with type 1 diabetes and disordered eating behaviour.

Methods and analysis This feasibility study will examine the effectiveness of a brief self-compassion intervention, compared with a waitlist control group. Participants aged 12-16 years will be recruited from three diabetes outpatient clinics in Auckland, New Zealand. The brief selfcompassion intervention is adapted from the standardised 'Making Friends with Yourself' intervention and will be delivered in a group format over two sessions. Apart from examining feasibility and acceptability through the flow of participants through the study and qualitative questions, we will assess changes to disordered eating behaviour (primary outcome), self-care behaviours, diabetes-related distress, self-compassion, stress and glycaemic control (secondary outcomes). Such data will be used to calculate the required sample size for a fully powered randomised controlled trial.

Ethics and dissemination This trial has received ethics approval from the Health and Disability Ethics Committee (research project number $\mathrm{A}+8467)$. Study results will be disseminated through peer-reviewed journals and conferences.

Trial registration number ANZCTR (12619000541101).

\section{INTRODUCTION}

This protocol paper describes a feasibility study designed to evaluate the feasibility, acceptability and estimate the effect of a brief self-compassion intervention for adolescents with type 1 diabetes (T1D) and disordered eating behaviours. Adolescents with T1D face
Strengths and limitations of this study

- The study will be the first to examine selfcompassion as a potential therapeutic approach to the widespread problem of disordered eating behaviour in adolescents with type 1 diabetes.

- Qualitative data, changes to self-reported psychological health, and $\mathrm{HbA1C}$ will be used to examine the programme's feasibility, acceptability and to estimate the magnitude of any effect of selfcompassion on disordered eating behaviour.

- The study is limited by the lack of blinding by the first author.

- The study is a feasibility trial and therefore will not be able to determine the efficacy of the brief selfcompassion programme.

- Overall, the study will contribute to the future research agenda of developing a 'clinically usable' self-compassion intervention for adolescents with type 1 diabetes.

the dual challenge of learning how to manage their chronic condition while concurrently dealing with what can be normatively difficult developmental changes. Maintaining optimal self-management of T1D requires adherence to a complicated routine of daily self-administration of insulin and monitoring diet, energy expenditure and blood glucose levels. ${ }^{1}$ Adolescents must learn how to make these complex routines flexible enough to implement into their daily life including school, hobbies and other activities. ${ }^{2}$ Unsurprisingly, diabetes self-management tends to deteriorate during adolescence, with adolescents showing sub-optimal glycaemic control and higher rates of complications compared with adults or younger children. ${ }^{3}$

Adolescents with T1D also show much greater rates of psychological disorders, especially eating disorders, than their healthy peers. ${ }^{45}$ In a recent national Australian study of adolescents with T1D, $50 \%$ of female 
and $18 \%$ of male adolescents scored over the cut-off for disordered eating behaviour. ${ }^{6}$ Eating disorders have also been shown to be twice as common in adolescent women with T1D compared with their healthy peers ${ }^{7}$ and rates of disordered eating in adolescents with T1D are rising. ${ }^{89}$ Given that behaviours such as insulin omission and purging are associated with increases in diabetesrelated complications, ${ }^{610}$ this is concerning. Poor psychological health, including disordered eating, has the potential to make daily self-management decisions even more challenging and has known associations with lower rates of glucose testing and insulin adherence, ${ }^{11}$ poorer glycaemic control ${ }^{12}$ and more frequent hospitalisations and complications. ${ }^{13}$ Therefore, prioritising psychological health and well-being is an essential component of successful diabetes care and may be even more relevant during adolescence.

Despite the high prevalence of mental health disorders among adolescents with T1D, treatment options are limited. Current interventions to target psychological issues include psychopharmacology (such as antidepressants) and psychosocial interventions. Research has shown that individual and group-based interventions focused on improving family relationships, stress management and coping skills can improve adherence, glycaemic control and quality of life. ${ }^{14}$ However, such interventions are rarely incorporated into standard diabetes care due to funding constraints and a lack of therapist availability, ${ }^{1516}$ as well as the logistical issues associated with family therapies. Furthermore, only one study has examined the use of an intervention specifically targeting disordered eating behaviour in adolescents with T1D. ${ }^{17}$ There is thus a strong need to develop interventions to treat psychological distress, especially disordered eating behaviour, that are acceptable to adolescents with T1D and can be realistically incorporated into routine clinical care.

Self-compassion is an emerging approach which may offer an effective method to improve psychological health and self-management among adolescents with T1D. Selfcompassion appears highly relevant to the self-criticisms that commonly arise following difficulty adhering to complex self-management regimens. At its core, selfcompassion is characterised by ${ }^{1}$ : being aware of one's moment to moment experiences with a sense of emotional balance and non-judgement (mindfulness), ${ }^{2}$ acknowledging that suffering and imperfection are part of being human (common humanity) and ${ }^{3}$ taking an active role in being caring and understanding towards oneself (selfkindness) ${ }^{18}$ Lower self-compassion has been closely linked to greater depression and anxiety, ${ }^{19}$ including in adults with diabetes, ${ }^{20} 21$ while higher self-compassion is associated with less rumination, self-criticism, perfectionism and fear of failure. ${ }^{1822}$ Self-compassion has demonstrated benefits for those with chronic illnesses (including inflammatory bowel disease and arthritis) such as improved selfmanagement and coping. ${ }^{23}$ Most recently, a standardised 8-week self-compassion programme for adolescents, 'Making Friends with Yourself' (MFY), adapted from the
Mindful Self-Compassion (MSC) programme for adults, ${ }^{24}$ showed significant improvements in self-compassion, life satisfaction and depression, compared with a waitlist control group, in healthy adolescents. ${ }^{23}$ Mindfulness, social connectedness, anxiety, stress, resilience, curiosity and gratitude have also been shown to increase throughout the MFY programme. ${ }^{25} 26$

To date, MFY has not been trialled in adolescents with diabetes or any other chronic illness. However, a recent randomised controlled trial (RCT) compared the 8-week MSC programme for adults with diabetes to a waitlist control group. ${ }^{21}$ Researchers found increased self-compassion was associated with reductions in diabetes-related distress, depression and improvements in glycaemic control, both at post-intervention and at the 3-month follow-up compared with the waitlist control group.

Considering the vital role psychological health plays in diabetes-related outcomes ${ }^{27}$ and the conceptual fit between the purpose of self-compassion training and the issues confronting adolescents with T1D, we developed a brief (2-session) self-compassion intervention targeted to adolescents with T1D and disordered eating behaviour. This study will examine the programme's feasibility and acceptability for adolescents with T1D and provide estimates of change in psychological and physical health outcomes. It is hypothesised that the brief selfcompassion intervention will improve disordered eating behaviours, self-care behaviours, diabetes-related distress, self-compassion, stress and glycaemic control, compared with the waitlist control group at post-intervention.

\section{METHODS AND ANALYSIS \\ Design overview}

The study is a feasibility study of a brief self-compassion intervention (treatment group), compared with a waitlist control group, in 28 adolescents with T1D. The study will be conducted in accordance with the Consolidated Standards of Reporting Trials (CONSORT) guidelines ${ }^{28}$ and has been prospectively registered in the Australian New Zealand Clinical Trials Registry. The Standard Protocol Items: Recommendations for Interventional Trials (SPIRIT) recommendations were adhered to in reporting the protocol. ${ }^{29}$

\section{Participants}

Participants in the study are adolescents with T1D who are currently being recruited from three paediatric and adolescent diabetes clinics in Auckland, New Zealand. Adolescents are eligible for inclusion in the study if they meet the following criteria ${ }^{1}$ : are aged $12-16$ years, ${ }^{2}$ diagnosed with T1D more than 6 months ago at time of recruitment and ${ }^{3}$ demonstrate moderate to high instances of disordered eating behaviour on the Diabetes Eating Problem Survey Revised (DEPS-R) ${ }^{30}$ screening tool. A moderate to high disordered eating behaviour cut-off score was chosen to allow more room to detect changes 
and to offer the interventions to those at the highest risk. Exclusion criteria include ${ }^{1}$ : non-English speaking adolescents, ${ }^{2}$ adolescents with developmental disorders (eg, autism spectrum disorder), ${ }^{3}$ adolescents diagnosed with a serious mental disorder requiring ongoing treatment (eg, psychosis), ${ }^{4}$ children with untreated hypothyroidism and ${ }^{5}$ children recently (in previous 48 hours) diagnosed with diabetic ketoacidosis (DKA) or severe hypoglycaemia. See figure 1 for an overview of the proposed flow of participants throughout the study.

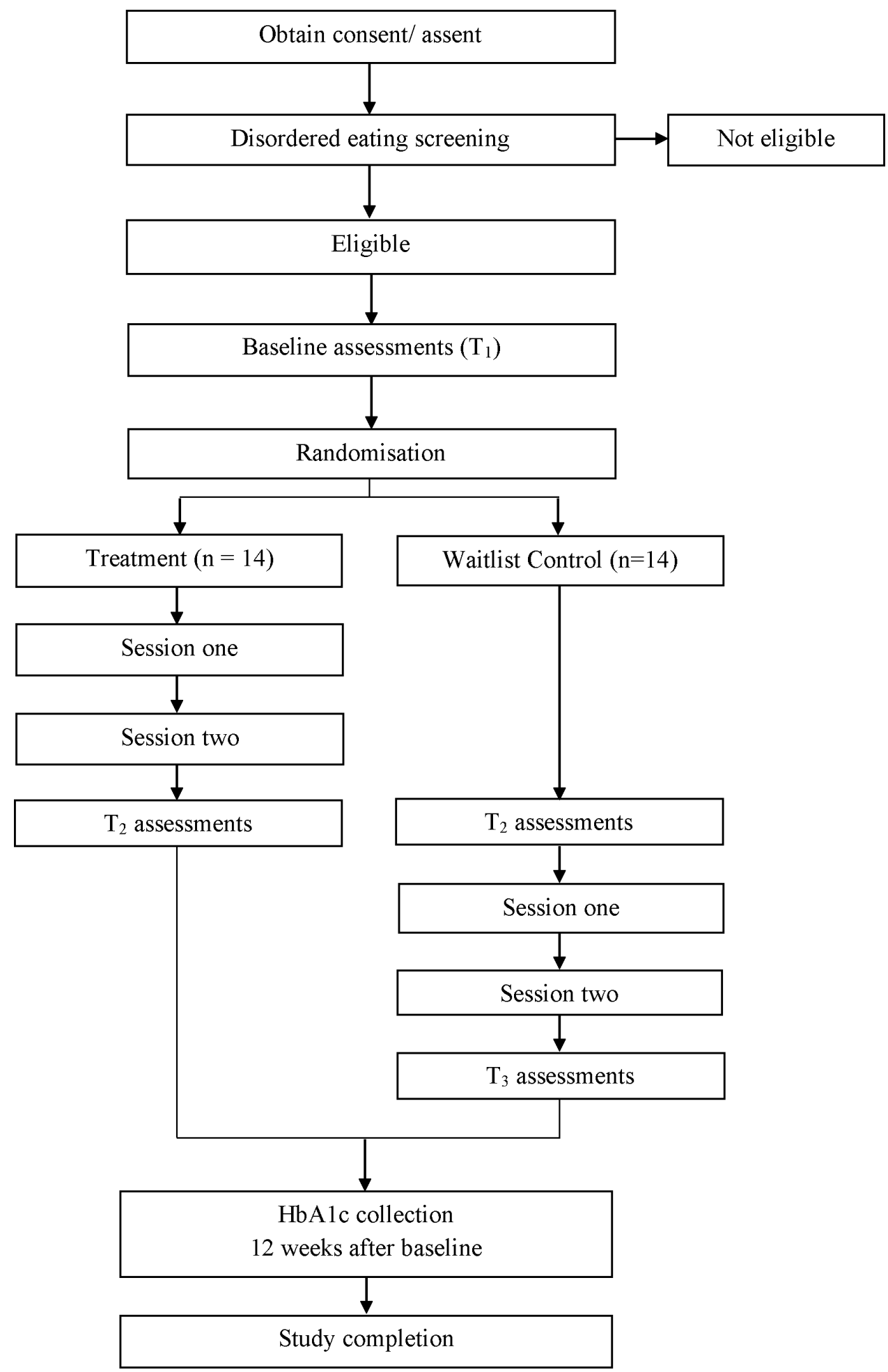

Figure 1 Consolidated Standards of Reporting Trials (CONSORT) flow diagram showing the proposed flow of participants $(n=28)$ through each stage of the feasibility trial (enrolment, disordered eating screening, baseline assessment, randomisation to either intervention group or waitlist control, time 2 assessment, time 3 assessment and collection of $\mathrm{HbA1c}$ ). 
Table 1 Key exercises and educational components of the brief self-compassion intervention for adolescents

\begin{tabular}{ll}
\hline Session $\mathbf{1}$ & Session $\mathbf{2}$ \\
\hline Topic: what is self-compassion? & Meditation: loving kindness meditation \\
\hline Mindful movement activity: stop and be meditation & Topic: summary of last week's content \\
\hline Topic: mindfulness and the wandering mind & Topic: self-esteems versus self-compassion \\
\hline Mindful observation activity: here-and-now stone & Discussion: the cost of social comparison \\
Topic: the adolescent brain & Meditation: compassionate body scan \\
Self-compassion exercise: how would I treat a friend? & Group exercise: crossing the line \\
\hline Self-compassion exercise: comforting gesture & Compassionate letter
\end{tabular}

Self-compassion exercise: three steps of self-compassion

Meditation: music meditation

\section{Sample size calculation}

Based on other studies suggesting the prevalence of disordered eating of $27 \%$ for boys and $42 \%$ for girls in T1D, ${ }^{31}$ we estimated that we would need to screen approximately 50 adolescents to enrol at least 20 adolescents (who score over the cut-off on the DEPS-R). We will use the observed changes in psychological outcomes (eg, DEPS-R) and physical health outcomes to calculate the required sample size for a subsequent fully powered RCT.

\section{Intervention development}

The brief self-compassion intervention was adapted from the 8-week MFY programme, ${ }^{25}$ in consultation with Karen Bluth and five other trained MFY and MSC teachers. Components from the MFY programme were chosen for the brief adaption because they $\operatorname{are}^{1}$ : foundational exercises covering the basics of mindfulness and self-compassion and ${ }^{2}$ address body image concerns and feelings of isolation, which have been highlighted in the literature to be relevant to adolescents with T1D. ${ }^{32}$ These components included group exercises and discussions, topics, art activities, meditations and individual reflection exercises (see table 1).

\section{Brief self-compassion intervention}

The brief self-compassion intervention will involve two 2-hour sessions conducted at The University of Auckland. The intervention sessions will be facilitated by the first author (AB), a trained MFY teacher.

As summarised in table 1 , session 1 will focus on giving participants an understanding of mindfulness through several tangible activities including a mindful movement activity, group discussions and a mindful activity using a stone or shell. Psychoeducation around adolescent brain development and emotion regulation systems ${ }^{33}$ will be taught to help to establish the reasons why mindfulness and self-compassion can help with managing stress, especially in adolescence. Regarding self-compassion, the 'How Would I Treat a Friend' exercise offers an interactive method of explaining self-compassion before practising the self-compassion coping skills of 'Comforting Gesture' (ie, 'Soothing Touch') and the 'Three Steps of Self-Compassion' (ie, 'A Moment for Me').
The 'How Would I Treat a Friend' exercise shows adolescents that they often treat themselves much more harshly than they treat their friends through exploring their reactions to how they would treat themselves versus their friends in difficult situations such as receiving a bad mark on an important test. Finding a 'Comforting Gesture' (such as holding your hands over your heart or stomach) involves practising and applying self-compassion to soothe and regulate emotions during stressful situations in one's life. The 'Three Steps of Self-Compassion' involves applying the three elements of self-compassion (mindfulness, common humanity and self-kindness) to cope with difficult emotions.

Session 2 is focused on developing coping skills to deal with body image concerns and feelings of isolation. Opening with a loving kindness meditation will provide a reminder of the concept of self-compassion and the 'Comforting Gesture' tool from session 1 before summarising the content taught in the last session. Selfesteem versus self-compassion and the cost of social comparison are then discussed to help teens understand how comparing themselves to others can create suffering and to allow them to practice using the coping skills taught in session 1 when they experience feelings of inadequacy. The 'Crossing the Line' group activity then emphasises the common humanity element of self-compassion by asking adolescents to cross a line of string if they have experienced certain feelings or situations such as feelings of isolation, been bullied or having compared themselves to their friends or an image in the media. Adolescents will then practice a compassionate body scan meditation and writing themselves a compassionate letter as further skills to use surrounding body image concerns.

Handbooks will be given to participants to review topics and outline the coping skills taught in the sessions, with examples of situations where it may be helpful to use them. Recordings of the meditations used in the sessions will also be emailed to the participant's parent/caregiver and the participant.

\section{Study procedure}

The study started recruitment on 24 May 2019 and is estimated to finish in late October 2019. Figure 1 depicts the 
flow of participants throughout the trial. Eligible families will be invited by diabetes nurse educators at their paediatric diabetes service clinic appointments to participate in a study investigating body image concerns in adolescents with T1D. Interested participants will be referred to the first author $(\mathrm{AB})$, who will verbally explain the study to interested families. Once consent/assent is given, the adolescents will complete the DEPS-R screening tool. Participants who score over the cut-off score for moderate disordered eating behaviour will then be randomised to either the treatment group or a waitlist control group (who will receive the intervention after the treatment group has completed post-intervention measures) on a 1:1 basis. Randomisation will occur via sealed envelopes labelled with sequential study numbers, prepared by a biostatistician independent of the study. As the first author $(\mathrm{AB})$ is responsible for both recruitment and teaching the programme, blinding is unable to occur.

After randomisation, participants will also complete standardised questionnaires measuring self-care behaviours, diabetes-related distress, self-compassion and stress in clinic or online. After completing the intervention, participants will be given the same questionnaires as well as open-ended questions to assess the acceptability and feasibility of the programme. Participants in the waitlist control will then start the intervention.

\section{Outcome measures}

The primary goal of this feasibility study is to determine the feasibility and acceptability of the brief selfcompassion intervention in the service of designing a larger, multi-centred, powered study. This will be assessed by evaluating whether adolescents with $\mathrm{T} 1 \mathrm{D}$ are willing to participate in the intervention (ie, recruitment and uptake), reasons for not wanting to participate, the acceptability of the delivered intervention (assessed via qualitative questions), study attrition rate and the suitability of the outcome measures. Qualitative data, through questionnaires at post-intervention, will also be used to refine the intervention content and comment on strengths and weaknesses of the programme.

In addition, the following psychological and physical health outcomes will be assessed to give preliminary estimates of effect sizes to estimate the sample size needed for a fully powered RCT:

1. Disordered eating behaviour as measured by the DEPS-R. ${ }^{30}$ The DEPS-R is a 16 -item diabetes-specific self-report measure of disordered eating. Participants are asked how true each item is for them (eg, 'I feel fat when I take all of my insulin') on a 6-point Likert scale $(0=$ never to $6=$ always $)$, with items summed to produce a total score. The scale has demonstrated good reliability $(\alpha=0.86-0.91)$ and validity in adolescents with diabetes samples. ${ }^{30} 3455$ For this study, the moderate disordered eating cut-off $(\geq 10)$ for inclusion in the study will be used. DEPS-R results for all adolescents screened for eligibility will be reported to give an indication of prevalence of moderate disordered eating behaviour in the paediatric diabetes clinics.

2. Self-care behaviours as measured by the Self Care Inventory-Revised Version (SCI-R) ${ }^{36}$ The SCI-R is a 15-item questionnaire which assesses how often diabetes self-care behaviours have been adhered to over the past $1-2$ months on a 5-point Likert scale $(1=$ never to $5=$ always). The self-care behaviours assessed include the main components of the T1D self-management routine such as monitoring and recording glucose levels, administering and adjusting insulin and regulating meals and exercise. Items will be averaged and converted to a $0-100$-point scale to produce a total score, with higher values representing better self-care. The scale has shown good reliability $(\alpha=0.77-0.78)$ and validity. ${ }^{37} 38$

3. Diabetes-related distress as measured by Problem Areas in Diabetes Questionnaire. ${ }^{39}$ The 20-item questionnaire lists common negative emotions related to living with diabetes such as, 'feeling alone with diabetes', and 'worrying about the future and the possibility of serious complications'. The 20-items are measured on a 6 -point Likert scale $(1=$ nota problem to $6=a$ serious problem), with items averaged to produce a total score. The scale demonstrates good reliability $(\alpha=0.93)$ and validity. ${ }^{40}$

4. Self-compassion as measured by the Self-Compassion Scale, short form. ${ }^{41}$ The 12 items are measured on a 5 -point Likert scale $(1=$ almost never to $5=$ almost always), with items averaged to create a total score. Example items include: "when I fail at something important to me I become consumed by feelings of inadequacy' and 'when I'm feeling down I tend to obsess and fixate on everything that's wrong'. Reliability for this scale is good with a Cronbach $\alpha=0.77-0.79^{25}{ }^{26}$ in adolescent samples. Strong predictive, convergent and discriminant validity has also been demonstrated. ${ }^{42} 43$

5. Stress as measured by the Perceived Stress Scale. ${ }^{44}$ The questionnaire assesses feelings of stress, hassles and coping during the past month. For example, 'in the last month, how often have you felt that you were unable to control the important things in your life?'. The 14-items are measured on a 5-point Likert scale $(0=$ never to $4=$ very often $)$, with the score of each item combined to produce a total score. The scale has been shown to associate with glycaemic control ${ }^{45}$ and demonstrates good reliability $(\alpha=0.75-0.88)$ and validity. ${ }^{46}$

6. Glycaemic control as measured by glycosylated haemoglobin $\left(\mathrm{HbA}_{1 \mathrm{C}}\right) \cdot \mathrm{HbA}_{1 \mathrm{C}}$ is a blood test collected during routine outpatient appointments (which occur every 3-4 months), which is a reliable measure of blood glucose control over a period of 3-4 months. $\mathrm{HbA}_{1 \mathrm{C}}$ is measured in millimoles per $\mathrm{mol}(\mathrm{mmol} / \mathrm{mol})$, with lower values indicating better glycaemic control. ${ }^{47}$ $\mathrm{HbA}_{1 \mathrm{C}}$ ranges from 31 to $108 \mathrm{mmol} / \mathrm{mol}$, with the ideal target for all child and adolescent age groups being below $58 \mathrm{mmol} / \mathrm{mol}^{1}{ }^{1} \mathrm{HbA}_{1 \mathrm{C}}$ levels will be accessed 
from clinical records at baseline and post-intervention (window of 12-16 weeks after baseline).

\section{Data analysis plan}

Descriptive statistics will be reported for proportion of adolescents who were screened and who scored over the cut-off for the DEPS-R, rates of disordered eating across all adolescents screened, number of adolescents who attended the sessions and number of adolescents who dropped out of the study. Data will be tested for violations of statistical assumptions. If parametric assumptions are not met, the Mann-Whitney test will compare the two groups at time 2 and Wilcoxon signed-rank test will analyse possible within-group improvements. Means, SD and $95 \%$ CI will be reported with the analyses. Pearson's correlations will be used to explore the relationships between the different outcome measures, demographic characteristics, disease characteristics and current insulin regimen. An independent sample t-test will be conducted at post-intervention (see time 2, figure 1) and will be our main analysis to test our hypothesis for differences between the intervention group and waitlist control group in disordered eating behaviour, diabetes-related distress, stress, self-care behaviours and self-compassion. An independent samples t-test will also be conducted at the 12-week follow-up to assess any possible differences between groups for glycaemic control. In addition, to increase sample size and statistical power, an exploratory analysis will combine postintervention data from participants in the treatment group (at time 2) and participants in the waitlist control group (at time 3) to examine within and between group changes from baseline, using paired sample t-tests. An intention to treat analysis is planned.

These effect size estimates will also allow us to estimate the required sample size for a future RCT. Mean differences, SD and sample size will be used to calculate an estimated Cohen's $d$ for the effect of the intervention on disordered eating behaviour, which we will then use to calculate our required sample size.

\section{Patient and public involvement}

Patients, carers and members of the public were not involved in the study design phases of this study. However, during the recruitment process patients who choose not to participate will be asked to assess whether the time commitment or required travel was a reason for not choosing to participate, when possible. In addition, patients who participate in the sessions will also provide qualitative feedback on the burden of participating, the recruitment process and the format and content of the sessions to inform the future RCT. Regarding dissemination of results, during recruitment we ask patients whether they consent to the findings being disseminated via peer-reviewed journal articles and conferences. We also ask whether they would like to be informed of the results at the end of the study and how (eg, email/post). Patients who agree to being informed of the study results are sent a letter with the key findings and any publications arising from the study. In some cases, patients may also be invited to a departmental presentation of the study findings.

\section{Ethics and dissemination}

Due to screening for and only including adolescents with disordered eating behaviour, it was recommended to include a waitlist control group (rather than a standard care control group) in order to offer all eligible participants the intervention. Participants who report significant psychological concerns, such as self-harm, or issues relating to their diabetes that fall outside of the scope of the intervention, such as complex self-management concerns, will be referred to the diabetes team psychologist. The paediatric and adolescent diabetes team (including endocrinologists, diabetes nurse educators, dietitians and a psychologist) will provide study support throughout the trial, including monitoring any adverse events. Study results will be disseminated through peerreviewed journals, a doctoral thesis and conference presentations.

\section{DISCUSSION}

To our knowledge, this study will be the first to assess the acceptability of self-compassion intervention for adolescents with T1D and examine the feasibility of a brief adaption of the MFY programme. The intervention addresses the rising concern of disordered eating in adolescents with $\mathrm{T} 1 \mathrm{D},{ }^{89}$ building on the sparse literature in this area. ${ }^{17}$ The intervention will aim to teach adolescents evidence-based mindfulness and self-compassion skills to help them cope with stress, difficult emotions, self-management obstacles and body image concerns and to determine whether self-compassion is a feasible and acceptable approach. As the prevalence and impact of these psychological concerns in adolescents with T1D are a current obstacle in diabetes care, the study will help determine whether self-compassion is a feasible and acceptable approach.

The novelty of testing self-compassion for adolescents with T1D and the brief format of the intervention are key strengths of this study. First, although self-compassion has previously demonstrated efficacy in adults with diabetes ${ }^{21}$ we believe it may be even more beneficial for adolescents with T1D. Adolescence is a period of vulnerability with an increased risk for self-criticism, poor self-esteem and body image concerns. ${ }^{48}$ In adolescents with T1D these concerns are exacerbated by difficult self-management routines and parental conflict surrounding their diabetes care ${ }^{49}$ as well as increased risk of psychological distress and feelings of isolation. ${ }^{32}$ Self-compassion may encourage adolescents to be more accepting and kind to themselves when facing these issues, without engaging in problematic or counterproductive self-criticism. The common humanity aspect of self-compassion is likely to help validate feelings that 
emerge from any struggles they are experiencing, such as feelings of isolation, feeling less than, stress and sadness, as normal and common to all teens.

An adapted version of the 'Making Friends with Yourself' programme to a brief (2-session format) was chosen to address the lack of 'clinically usable' interventions in diabetes care. Although it is widely acknowledged that psychological care is a key component of improving health outcomes in diabetes, ${ }^{1627}$ the integration of psychosocial care into routine diabetes care remains lacking. Despite the growing literature demonstrating the efficacy of psychosocial interventions for improving psychological outcomes in T1D, interventions are rarely designed with the intention of being 'clinically usable' and in a format that could potentially be adopted into standard care.

Altogether, our aim is to examine the feasibility and acceptability of a brief self-compassion intervention, as well as provide effect size estimates. If the brief selfcompassion intervention is found to be feasible and acceptable to adolescents, future plans will include conducting a fully powered RCT to examine the longterm efficacy of the intervention, with the intention of developing a clinically usable psychosocial intervention for youth with both T1D and disordered eating behaviours.

\section{Twitter Anna L Boggiss @anna_boggiss}

Contributors ALB, PLH and ASS came up with the conception and design of the study. ALB will be recruiting participants and conducting the program sessions. PLH and CJ will provide guidance and assistance with recruitment. KB provided training and consultation during the program development phase and will provide ALB with supervision throughout the sessions. ALB and ASS wrote the manuscript. NSC, PLH, $\mathrm{CJ}$ and $\mathrm{KB}$ reviewed and edited the manuscript.

Funding The authors have not declared a specific grant for this research from any funding agency in the public, commercial or not-for-profit sectors.

Competing interests None declared.

Patient consent for publication Not required.

Provenance and peer review Not commissioned; externally peer reviewed.

Open access This is an open access article distributed in accordance with the Creative Commons Attribution Non Commercial (CC BY-NC 4.0) license, which permits others to distribute, remix, adapt, build upon this work non-commercially, and license their derivative works on different terms, provided the original work is properly cited, appropriate credit is given, any changes made indicated, and the use is non-commercial. See: http://creativecommons.org/licenses/by-nc/4.0/.

ORCID iDs

Anna L Boggiss http://orcid.org/0000-0002-7336-955X

Nathan S Consedine http://orcid.org/0000-0002-7691-0938

\section{REFERENCES}

1 American Diabetes Association. Standards of Medical Care in Diabetes - 2017 Abridged for Primary Care Providers. Clinical Diabetes 2017;35:5-26.

2 Chiang JL, Kirkman MS, Laffel LMB, et al. Type 1 diabetes through the life span: a position statement of the American diabetes association. Diabetes Care 2014;37:2034-54.

3 Moore SM, Hackworth NJ, Hamilton VE, et al. Adolescents with type 1 diabetes: parental perceptions of child health and family functioning and their relationship to adolescent metabolic control. Health Qual Life Outcomes 2013;11:50.
4 Reynolds KA, Helgeson VS. Children with diabetes compared to Peers: depressed? distressed? A meta-analytic review. Annals of Behavioral Medicine 2011;42:29-41.

5 Young V, Eiser C, Johnson B, et al. Eating problems in adolescents with Type 1 diabetes: a systematic review with meta-analysis. Diabetic Medicine 2013;30:189-98.

6 Ackard DM, Vik N, Neumark-Sztainer D, et al. Disordered eating and body dissatisfaction in adolescents with type 1 diabetes and a population-based comparison sample: comparative prevalence and clinical implications. Pediatr Diabetes 2008;9:312-9.

7 Jones JM, Lawson ML, Daneman D. Eating disorders in adolescent females with and without type 1 diabetes: cross sectional study. BMJ 2000;320:1563-6.

8 Young-Hyman DL, Davis CL. Disordered eating behavior in individuals with diabetes: importance of context, evaluation, and classification. Diabetes Care 2010;33:683-9.

9 Colton PA, Olmsted MP, Daneman D, et al. Five-Year prevalence and persistence of disturbed eating behavior and eating disorders in girls with type 1 diabetes. Diabetes Care 2007;30:2861-2.

10 Neumark-Sztainer D, Patterson J, Mellin A, et al. Weight contro practices and disordered eating behaviors among adolescent females and males with type 1 diabetes: associations with sociodemographics, weight concerns, familial factors, and metabolic outcomes. Diabetes Care 2002;25:1289-96.

11 Borus JS, Laffel L. Adherence challenges in the management of type 1 diabetes in adolescents: prevention and intervention. Curr Opin Pediatr 2010;22:405-11.

12 Bernstein CM, Stockwell MS, Gallagher MP, et al. Mental health issues in adolescents and young adults with type 1 diabetes: prevalence and impact on glycemic control. Clinical Pediatrics 2013;52:10-15.

13 Stewart SM, Rao U, Emslie GJ. Depressive symptoms predict hospitalization for adolescents with type 1 diabetes mellitus. Pediatrics 2005;115:1315-9.

14 Delamater AM, de Wit M, McDarby V, et al. Psychological care of children and adolescents with type 1 diabetes. Pediatr Diabetes 2014;15:232-44.

15 Hampson SE, Skinner TC, Hart J, et al. Behavioral interventions for adolescents with type 1 diabetes: how effective are they? Diabetes Care 2000;23:1416-22.

16 Hilliard ME, Powell PW, Anderson BJ. Evidence-Based behavioral interventions to promote diabetes management in children, adolescents, and families. American Psychologist 2016;71:590-601.

17 Olmsted MP, Daneman D, Rydall AC, et al. The effects of psychoeducation on disturbed eating attitudes and behavior in young women with type 1 diabetes mellitus. Int J Eat Disord 2002;32:230-9.

18 Neff K. Self-compassion: an alternative conceptualization of a healthy attitude toward oneself. Self and Identity 2003;2:85-101.

19 Marsh IC, Chan SWY, MacBeth A. Self-compassion and psychological distress in adolescents - a meta-analysis. Mindfulness 2018;9:1011-27.

20 Friis AM, Johnson MH, Cutfield RG, et al. Does kindness matter? Self-compassion buffers the negative impact of diabetes-distress on $\mathrm{HbA}$. Diabetic Medicine 2015;32:1634-40.

21 Friis AM, Johnson MH, Cutfield RG, et al. Kindness Matters: A Randomized Controlled Trial of a Mindful Self-Compassion Intervention Improves Depression, Distress, and $\mathrm{HbA}_{1 \mathrm{c}}$ Among Patients With Diabetes. Diabetes Care 2016;39:1963-71.

22 Neff KD, Hsieh Y-P, Dejitterat K. Self-compassion, achievement goals, and coping with academic failure. Self and ldentity 2005;4:263-87.

23 Sirois FM, Molnar DS, Hirsch JK. Self-compassion, stress, and coping in the context of chronic illness. Self and Identity 2015;14:334-47.

24 Neff KD, Germer CK. A pilot study and randomized controlled trial of the mindful Self-Compassion program. J Clin Psychol 2013;69:28-44.

25 Bluth K, Eisenlohr-Moul TA. Response to a mindful self-compassion intervention in teens: a within-person association of mindfulness, self-compassion, and emotional well-being outcomes. J Adolesc 2017;57:108-18.

26 Bluth K, Gaylord SA, Campo RA, et al. Making friends with yourself: a mixed methods pilot study of a mindful self-compassion program for adolescents. Mindfulness 2016;7:479-92.

27 Young-Hyman D, de Groot M, Hill-Briggs F, et al. Psychosocial care for people with diabetes: a position statement of the American diabetes association. Diabetes Care 2016;39:2126-40.

28 Moher D, Hopewell S, Schulz KF, et al. Consort 2010 explanation and elaboration: updated guidelines for reporting parallel group randomised trials. BMJ 2010;340:c869. 
29 Chan A-W, Tetzlaff JM, Altman DG, et al. Spirit 2013 statement: defining standard protocol items for clinical trials. Ann Intern Med 2013;158:200-7.

30 Markowitz JT, Butler DA, Volkening LK, et al. Brief screening tool for disordered eating in diabetes: internal consistency and external validity in a contemporary sample of pediatric patients with type 1 diabetes. Diabetes Care 2010;33:495-500.

31 Cherubini V, Skrami E, lannilli A, et al. Disordered eating behaviors in adolescents with type 1 diabetes: a cross-sectional populationbased study in Italy. Int J Eat Disord 2018;51:890-8.

32 Storch EA, Ledley DR. Peer victimization and psychosocial adjustment in children: current knowledge and future directions. Clin Pediatr 2005;44:29-38.

33 Gilbert P. Compassion: Conceptualisations, research and use in psychotherapy. Routledge, 2005

34 Araia E, Hendrieckx C, Skinner T, et al. Gender differences in disordered eating behaviors and body dissatisfaction among adolescents with type 1 diabetes: results from diabetes miles youthAustralia. Int J Eat Disord 2017;50:1183-93.

35 Wisting L, Frøisland DH, Skrivarhaug T, et al. Psychometric properties, norms, and factor structure of the diabetes eating problem Survey-Revised in a large sample of children and adolescents with type 1 diabetes. Diabetes Care 2013;36:2198-202.

36 Weinger K, Butler HA, Welch GW, et al. Measuring diabetes self-care: a psychometric analysis of the self-care Inventory-revised with adults. Diabetes Care 2005;28:1346-52.

37 Khagram L, Martin CR, Davies MJ, et al. Psychometric validation of the self-care Inventory-Revised (SCI-R) in UK adults with type 2 diabetes using data from the AT.LANTUS follow-on study. Health Qual Life Outcomes 2013;11:24.

38 Vaala SE, Hood KK, Laffel L, et al. Use of commonly available technologies for diabetes information and self-management among adolescents with type 1 diabetes and their parents: a web-based survey study. Interact J Med Res 2015;4:e24.

39 Polonsky WH, Anderson BJ, Lohrer PA, et al. Assessment of diabetes-related distress. Diabetes Care 1995;18:754-60.

40 Schmitt A, Reimer A, Kulzer B, et al. How to assess diabetes distress: comparison of the problem areas in diabetes scale (paid) and the diabetes distress scale (DDS). Diabetic Medicine 2016;33:835-43.

41 Raes F, Pommier E, Neff KD, et al. Construction and factorial validation of a short form of the Self-Compassion scale. Clin Psychol Psychother 2011;18:250-5.

42 Neff KD. The development and validation of a scale to measure selfcompassion. Self and Identity 2003;2:223-50.

43 Neff KD, Rude SS, Kirkpatrick KL. An examination of selfcompassion in relation to positive psychological functioning and personality traits. J Res Pers 2007;41:908-16.

44 Cohen S, Kamarck T, Mermelstein R. A global measure of perceived stress. J Health Soc Behav 1983;24:385-96.

45 Surwit RS, van Tilburg MAL, Zucker N, et al. Stress management improves long-term glycemic control in type 2 diabetes. Diabetes Care 2002;25:30-4.

46 Lee E-H. Review of the psychometric evidence of the perceived stress scale. Asian Nurs Res 2012;6:121-7.

47 Daneman D. Type 1 diabetes. The Lancet 2006;367:847-58.

48 Neff KD, McGehee P. Self-compassion and psychological resilience among adolescents and young adults. Self and Identity 2010;9:225-40.

49 Serlachius A, Northam E, Frydenberg E, et al. Adapting a generic coping skills programme for adolescents with type 1 diabetes: a qualitative study. J Health Psychol 2012;17:313-23. 